\title{
Pax3 is required for enteric ganglia formation and functions with Sox10 to modulate expression of $c$-ret
}

\author{
Deborah Lang, Fabian Chen, Rita Milewski, Jun Li, Min Min Lu, and Jonathan A. Epstein \\ Cardiovascular Division, Department of Medicine, University of Pennsylvania, Philadelphia, Pennsylvania, USA
}

Address correspondence to: Jonathan A. Epstein, 954 BRB II, 421 Curie Boulevard, Philadelphia, Pennsylvania 19104, USA. Phone: (215) 898-8731; Fax: (215) 573-2094; E-mail: epsteinj@mail.med.upenn.edu.

Deborah Lang and Fabian Chen contributed equally to this work.

Received for publication July 20, 2000, and accepted in revised form September 5, 2000.

\begin{abstract}
Hirschsprung disease and Waardenburg syndrome are human genetic diseases characterized by distinct neural crest defects. Patients with Hirschsprung disease suffer from gastrointestinal motility disorders, whereas Waardenburg syndrome consists of defective melanocyte function, deafness, and craniofacial abnormalities. Mutations responsible for Hirschsprung disease and Waardenburg syndrome have been identified, and some patients have been described with characteristics of both disorders. Here, we demonstrate that $P A X 3$, which is often mutated in Waardenburg syndrome, is required for normal enteric ganglia formation. Pax 3 can bind to and activate expression of the $c-R E T$ gene, which is often mutated in Hirschsprung disease. Pax3 functions with Sox10 to activate transcription of $c-R E T$, and SOX10 mutations result in Waardenburg-Hirschsprung syndrome. Thus, Pax3, Sox10, and c-Ret are components of a neural crest development pathway, and interruption of this pathway at various stages results in neural crest-related human genetic syndromes.
\end{abstract}

J. Clin. Invest. 106:963-971 (2000).

\section{Introduction}

Neural crest cells arise from the dorsal neural tube and migrate over precise pathways to populate multiple organs and tissues in the developing embryo (1). They differentiate into many cell types including melanocytes, neurons, and glia. In humans, various syndromes have been described that are characterized by deficiencies of neural crest derivatives (2). In recent years, some of the genes responsible for these disorders have been identified, and relationships among these genes and syndromes have begun to emerge.

A subset of neural crest precursors gives rise to the enteric nervous system (3). The intestine is colonized by a rostral to caudal wave of migrating cells originating from the vagal crest region, although truncal and sacral crest cells also contribute. Enteric ganglia precursors must both migrate and proliferate in order to provide the necessary expansion of the precursor pool required for the population of the entire intestinal tract. This process is critically dependent on several growth factors and their cognate receptors. For instance, enteric ganglia precursors express the c-Ret tyrosine kinase receptor that is required for their survival and proliferation (4-7). The ligand for c-Ret, glial cell-derived neurotrophic factor (GDNF), is expressed by mesenchymal cells surrounding enteric ganglia and is required for proper enteric plexus development (8-11). The endothelin B receptor (EDNRB) and its ligand endothelin 3 (EDN3) are also required (12-16), suggesting that multiple growth factor pathways converge to regulate enteric neuron development and mat- uration. In addition, several transcription factors, including Sox10, Phox2b, and Mash1 regulate various aspects of enteric neuron differentiation (17-23).

In humans, abnormalities of the enteric nervous system result in Hirschsprung disease (aganglionic megacolon) and gastrointestinal motility disorders. The most common mutations found in patients with Hirschsprung disease are in the $c-R E T$ gene $(5,6)$. Families with Hirschsprung disease have also been described in which heterozygous mutations in $E D N R B$ or $E D N 3$ have been identified $(14,16,24)$. Interestingly, homozygous mutations of either of these genes result in additional phenotypes characteristic of Waardenburg syndrome that include pigmentation abnormalities, craniofacial defects, and deafness $(16,24,25)$.

Heterozygous mutations in SOX10 also result in features of both Waardenburg syndrome and Hirschsprung disease (19). Sox10 is a member of the high-mobility group (HMG) of nuclear factors related to the sex-determining factor Sry (26). Sox10 is capable of sequence-specific DNA binding (26), whereas HMG proteins in general function as transcriptional coactivators by acting as architectural proteins and synergizing with other transcriptional regulators $(27,28)$. Mutations in Sox10 in the mouse account for the Dom phenotype that has been studied as a model for Hirschsprung disease (20).

Patients with Waardenburg syndrome without features of Hirschsprung disease most often harbor mutations in the $P A X 3$ gene $(29,30)$. Pax3 is a member of the paired-box-containing family of nuclear 
transcription factors (31). Affected individuals are heterozygous for $P A X 3$ mutations; homozygous loss of function is lethal (32). Waardenburg syndrome can also be caused by mutations in the MITF gene (33), and Pax3 probably directly regulates MITF transcription during melanocyte development (34). Enteric ganglia defects have not been reported in patients with Waardenburg syndrome with $P A X 3$ or MITF mutations.

In the mouse, $P a x 3$ mutations result in the Splotch phenotype $(35,36)$, characterized by a white belly spot in the heterozygous state (37). Homozygous-deficient embryos die during midgestation with neural tube and cardiac defects. Abnormalities of the enteric nervous system have not been previously identified.

Here, we demonstrate that Pax3-expressing neural crest precursors give rise to enteric ganglia. We show that Pax3 is required for formation of the enteric nervous system. Furthermore, Pax3 synergizes with Sox10 to activate an enhancer in the c-RET gene, and Pax3 and Sox10 bind to adjacent sites within this enhancer. Pax3, Sox10, and c-Ret are components of a molecular cascade regulating neural crest and enteric nervous system development.

\section{Methods}

Transgenic mice. P3pro-Cre consisted of the $1.6-\mathrm{kb}$ proximal Pax3 regulatory region upstream of Cre recombinase (38). RET-lacZ was generated by replacement of a HindIII-BamHI fragment of RET-luc (see below) containing the luciferase gene with a HindIII-BamHI fragment from $\mathrm{pSV}-\beta$-gal (Promega Corp., Madison, Wisconsin, USA) encoding $\beta$-galactosidase. Splotch mice and embryos were genotyped as described elsewhere (39).

Immunobistochemistry. Immunohistochemistry for detection of neural cell specific neurofilament was performed on paraffin-embedded sections of tissue fixed in $4 \%$ paraformaldehyde after exposing antigen with Antigen Unmasking Solution (Vector Laboratories, Burlingame, California, USA) and incubation with $2 \mathrm{H} 3$ $\mathrm{mAb}$ (Developmental Studies Hybridoma Bank, Iowa City, Iowa, USA) diluted 1:200. Antibody was detected using either peroxidase or FITC conjugated goat antimouse secondary antibody. Antibodies to c-Ret (Immuno-Biological Laboratories, Tokyo, Japan), neuron-specific enolase (DAKO Corp., Carpinteria, California, USA), GFAP (DAKO Corp.), and S100 (Novocastra, Newcastle-upon-Tyne, United Kingdom) were used at dilutions of 1:50, 1:200, 1:500, and 1:200, respectively. Paraformaldehyde-fixed (10 minutes) frozen sections were used with the c-Ret antibody and paraffin sections of paraformaldehyde-fixed ( 24 hours) tissue were used for the other antibodies.

In situ bybridization. Radioactive (40) and whole mount (41) in situ hybridization studies were performed as described elsewhere. Specific sequences used as templates for synthesis of RNA probes are listed as follows with gene name followed by GenBank accession number and specific base pairs amplified: $c$ - ret, X67812, bp 2142-2608; Sox10, AF047043, bp 1151-1450; ednrb, U32329, bp 1506-1794; edn3, U32330, bp 737-1041; SM22 $\alpha$, L41154, bp 696-975.

Northern blot analysis. RNA was prepared 48 hours after transfection of P19 cells, denatured with glyoxal and dimethyl sulfoxide, electrophoresed on a $1.5 \%$ agarose/0.01M phosphate buffer gel, transferred to a nylon membrane (Genescreen Plus; NEN Life Science Products, Boston, Massachusetts, USA), and hybridized with a $c$-ret probe (bp 2142-2608 of Genbank no. X67812).

Luciferase assays. NIH 3T3 and 293T cells were maintained in DME with 10\% FCS and transfected at 50\% confluency in $35-\mathrm{mm}$ dishes. A total of $2 \mu \mathrm{g}$ of plasmid DNA was mixed with $40 \mu \mathrm{l}$ Effectene reagent and used to transfect quadruplicate wells (QIAGEN Inc., Valencia, California, USA). Each reaction, used for four equal transfections, contained $0.5 \mu \mathrm{g}$ of reporter/luciferase plasmid, $0.5 \mu \mathrm{g}$ of $\mathrm{pCMV}-\beta$-galactosidase, and variable amounts of pCMV-Pax3 (42), pCMV-Pax 2 (provided by R. Maas), pCMV-Pax6 (provided by R. Maas), pCDNA-Pax9 (derived from pcPax9K5, provided by $H$. Peters), and/or pCMV-Sox 10 (derived from dcgs10-1, provided by M. SouthardSmith and W. Pavan). Total amount of DNA was kept constant by the addition of pBS plasmid. After 72 hours, luciferase assays (Promega Corp.) and $\beta$-galactosidase assays using ONTG substrate (Promega Corp.) were performed. Luciferase activity was normalized for $\beta$-galactosidase transfection efficiency. RET-luc was prepared by PCR using forward primer 5'CCGCTCGAGCTTCCACTCTCCACGTTGCTT and reverse primer 5'-CCCAAGCTTCGGTCGCGGGACTGAAGCTAG with TaKaRa DNA polymerase (PanVera, Madison, Wisconsin, USA). The PCR product was cloned into the XhoI and HindIII sites of pGL2-basic (Promega Corp.). Initial transfections determined the optimal ratios of expression plasmid to reporter plasmid and established a dose-response curve (data not shown). At high concentrations, Pax3 inhibited luciferase reporter activity, as has been described previously (43).

Deletion constructs derived from RET-luc were prepared by restriction digestion and religation, or by PCR amplification and replacement of the NheI-NcoI fragment of construct D15. The composition of each construct follows; all RET promoter base pair numbering corresponds to Genbank accession number AF032124. D1 (938-5100), D14 (1-1695, 4050-5100), D15 (938-1695, 4050-5100), D4 (2460-5100), D7 (4047-5100), D9 (4910-5100). The following deletion constructs contain the proximal promoter (bp 4050$5100)$ in addition to sequences listed: 615 (10801695), 570 (1080-1650), 550 (1099-1650). Construct 615 mut (1080-1695) was generated with a reverse primer that replaced the putative Pax3 binding site with an AscI restriction site (underlined): 5'-CAT GCA TGG GGG CGC GCC TAT GGT GGT TGG AAG CAC AGA CTC TG. All fragments generated by PCR were subcloned into the NheI and NcoI sites of construct D15. 
Electrophoretic mobility shift assay. Electrophoretic mobility shift assay (EMSA) was performed as described previously (44) in the presence of $250 \mu \mathrm{g} / \mathrm{ml}$ poly-dIdC and $50 \mathrm{mM} \mathrm{KCl}$. Nonradioactive competitor DNA, when included, was present at 100-fold molar excess over labeled probe. The Pax3 protein used was GST-P3PdHd (45) that includes the Pax3 paired and homeodomains. In vitro transcribed and translated Sox10 (TNT kit, Promega) was prepared from ClaI linearized Sox10 plasmid (dgs10-1) as template. Products of control in vitro transcription/translation reactions performed in the absence of Sox 10 plasmid resulted in no detectable electrophoretic mobility shift with any probe used. Primers used for EMSA are listed as follows; engineered mutations are underlined. Probe A: 5'-CAT GCA GAG TCT GTG CTT CCA ACC ACC ATG TCA CAC TGC CCA TG; APaxmut: 5'CAT GCA GAG TCT GTG CTT CCA ACC ACC ATA GGC GCG CCC CCA TG; ASoxmut: 5'-CAT GCA GAG TGG CGC GCC CCA ACC ACC ATG TCA CAC TGC CCA TG.

\section{Results}

Pax3-expressing precursors are fated to become enteric ganglia. We have recently demonstrated that the proximal $1.6-\mathrm{kb}$ upstream regulatory region of the murine $P a x 3$ gene is sufficient to direct transgene expression to the dorsal neural tube and to neural crest cells (46). This transgene recapitulates a subdomain of endogenous Pax3 expression (31) and omits expression in all myogenic precursors. We have created transgenic mice using the 1.6-kb Pax3 regulatory element directing Cre recombinase expression in neural crest progenitors so that we could determine the fate of Pax3 expressing neural crest cells $(38,47,48)$. The pattern of Cre expression in these mice is consistent with the previously characterized activity of the Pax3 promoter element used $(46,49)$, which directs expression that initiates at approximately embryonic day 9 (E9) and is restricted to dorsal neural tube and neural crest. Transgenic embryos resulting from a cross to R26R Cre reporter mice in which $\beta$-galactosidase is constitutively expressed only in cells that are subjected to Cremediated recombination (48) demonstrate prominent $\beta$-galactosidase activity at E14.5 in the stomach and intestine (Figure 1a). Cross sections through labeled loops of intestine indicate that cells expressing $\beta$-galactosidase represent enteric ganglia (Figure 1b, arrows) and express neurofilament (Figure 1, $\mathrm{c}$ and d). We utilized three independent transgenic Cre lines in these and subsequent experiments to ensure that transgene insertion sites were not confounding results. Pax3 expression is not detectable in enteric neurons between E11.5 and E16.5, suggesting that Pax3 functions in migrating or premigratory cells. Our fate mapping data indicate that enteric ganglia are among the tissues derived from Pax3-expressing neural crest cells.

Pax3 is required for normal enteric ganglia development. We have confirmed and extended these results using a different transgenic mouse that also expresses $\beta$-galactosidase in neural crest derivatives and we have used this transgenic line to track neural crest cell fate in Pax3-deficient Splotch embryos. The 6.5 -kb proximal connexin $43(\mathrm{C} \times 43)$ promoter directs expression in a subset of the endogenous $C \times 43$ expression domain that includes dorsal neural tube, dorsal root ganglia, cardiac neural crest, and other neural crest-derived tissues (50). a

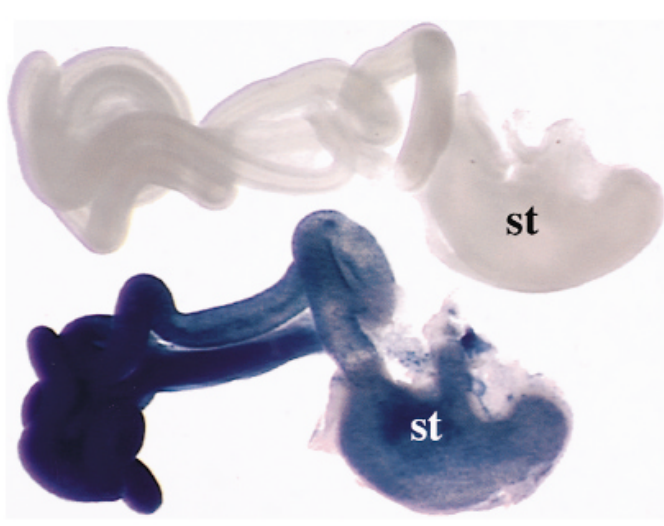

b

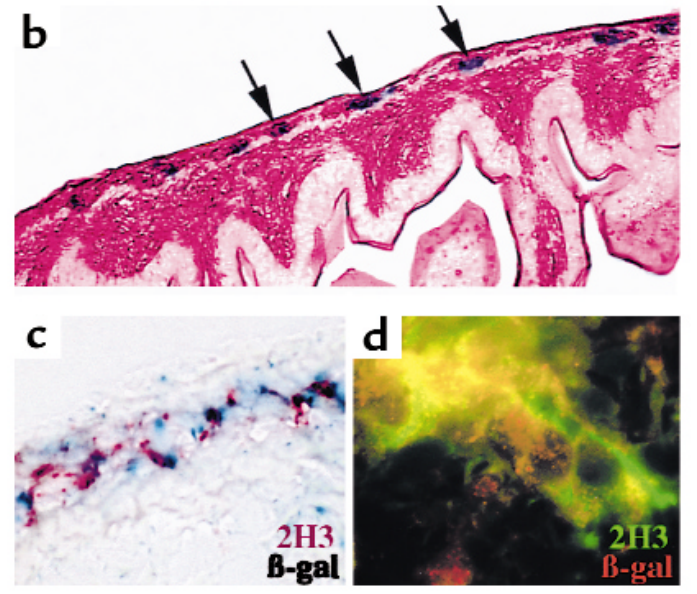

\section{Figure 1}

A subset of Pax3-expressing cells are fated to become enteric ganglia. Neural crest-specific regulatory elements of the Pax3 promoter were used to make transgenic mice expressing Cre recombinase. (a) After mating with Cre reporter mice, Pax3-expressing neural crest cells express $\beta$-galactosidase (stained blue) and populate the stomach (st) and intestines. The gastrointestinal tract from an E14.5 transgenic embryo is blue (bottom), whereas tissue from a Cre reporter embryo without the P3pro-Cre transgene processed in parallel is not stained (top). (b) Transverse section through a labeled loop of bowel reveals $\beta$-galactosidase-expressing cells in the region populated by enteric ganglia (arrows). The section is counterstained with eosin. (c) Coimmunohistochemistry of an adjacent section to that shown in b reveals neurofilament expression ( $2 \mathrm{H} 3$ antibody, red) colocalizing with $\beta$-galactosidase (blue). (d) Higher-power image of a similar section to that shown in c colabeled for neurofilament $(2 \mathrm{H} 3$, green) and $\beta$-galactosidase (red) expression. Colocalization results in a yellow signal, identifying labeled cells as enteric ganglia. 
We have crossed Cx43-lacZ transgenic mice to Splotch mice, and we have examined enteric ganglia development at E10.5-E12.5. Litters from pregnant $S p^{+/-}$ females derived from matings with transgenic $S p^{+/-}$ males were stained for $\beta$-galactosidase activity. In wildtype embryos, positively staining cells were seen lining the foregut at E10.5 (Figure 2a) and formed a confluent ring encircling each loop of bowel by E12.5 (Figure 2c). In $S p^{-/-}$embryos, few positively staining cells were seen surrounding the gut at E10.5 (Figure 2b). At E12.5, some labeled cells were identified in the stomach of Splotch embryos, but few or no labeled cells were seen in the intestine distal to the stomach (Figure 2d). Enteric ganglia are thought to populate the gut in a

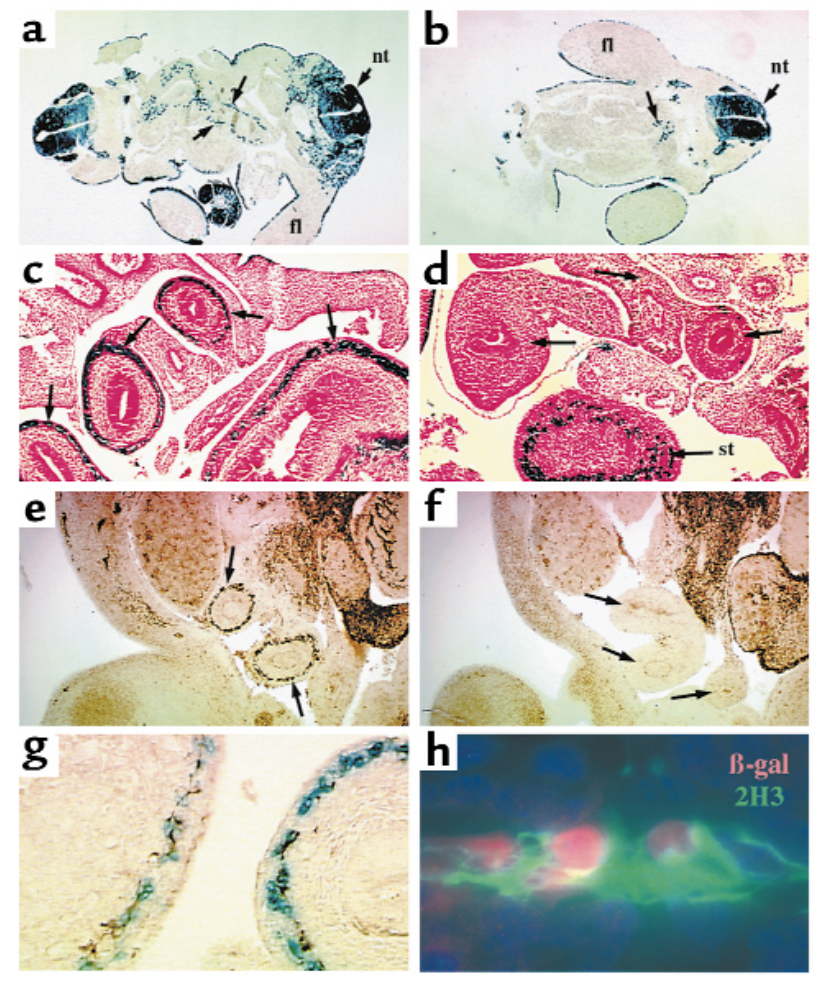

Figure 2

Enteric ganglia are deficient in Splotch intestine. ( $\mathbf{a}$ and $\mathbf{b})$ Transverse sections of E10.5 wild-type (a) and $S p^{-1-}$ (b) Cx43-LacZ transgenic embryos are shown after staining for $\beta$-galactosidase activity (blue). $\beta$-Galactosidase-positive cells are seen lining the primitive gut in wildtype (arrows, a) but not $\mathrm{Sp}^{-/-}$(b) embryos, although a small number of blue cells are seen near the proximal foregut (arrow, b). (c and d) $\beta$-Galactosidase detection in E12.5 wild-type (c) and $\mathrm{Sp}^{-/-}$(d) transverse sections at the abdominal level reveals expression in the wall of the stomach (st) and all loops of bowel (arrows, c) in wild-type embryos. No expression is seen in the gut distal to the stomach in $S p^{-/-}$embryos (arrows, d). (e and f) Neurofilament immunohistochemistry of E12.5 wild-type (e) and $\mathrm{Sp}^{-1-}$ (f) sections shows loss of neurofilament expression in $\mathrm{Sp}^{-/-}$embryos in a pattern similar to that seen for $\beta$-galactosidase (compare arrows, e and $\mathbf{f}$ ). (g) Costaining for $\beta$-galactosidase (blue) and neurofilament (brown) shows overlapping expression patterns in the bowel wall of wild-type embryos. (h) Higher power of nuclear $\beta$-galactosidase (red) and neurofilament ( $2 \mathrm{H} 3$, green) stained wild-type intestine confirms that Cx43-lacZ transgene labels enteric neurons. nt, neural tube. $\mathrm{fl}$, forelimb. rostral to caudal wave of migration. Our results in Splotch embryos suggest delayed or deficient neural crest colonization of the developing intestine.

We confirmed that differences in transgene expression in $S p^{-/-}$versus wild-type embryos represented differences in enteric ganglia formation by costaining for expression of neural cell-specific neurofilament using a mAb (2H3). Neurofilament expression was similar to Cx43 transgene expression in the intestinal wall of wild-type embryos (Figure 2e) and was deficient in Splotch embryo intestines (Figure 2f). Costaining of wild-type tissue with antibodies specific for $\beta$-galactosidase and neurofilament demonstrated colocalization (Figure 2, $\mathrm{g}$ and $\mathrm{h}$ ), confirming transgene expression by enteric ganglia cells.

The c-Ret tyrosine kinase receptor is required for enteric ganglia development in the intestine (5-7). At E9.5 and E10.0, whole mount in situ hybridization reveals c-ret-expressing cells (Figure 3a). In Splotch embryos, c-ret expression is reduced (Figure 3a). By E12.5, c-ret is expressed in the bowel wall of wild-type embryos (WT, Figure 3, b and c) in a pattern similar to Cx43-lacZ and neurofilament. Expression of c-ret at the RNA (Figure 3f) and protein (Figure 3g) level is deficient in $S p^{-/-}$embryos, although some expression is retained in the stomach (st, Figure 3f). Immunohistochemistry using antibodies specific for neuron specific enolase and for glial markers GFAP and S-100 showed similar differences between wild-type and Splotch embryos, suggesting that most or all neural crest derivatives in the intestine are affected by the absence of Pax3 (see Methods; data not shown).

Cell-autonomous function for Pax3 in enteric ganglia precursors. Given that expression and fate mapping data indicate that Pax3 is required for enteric ganglia formation, we asked whether this represented a cellautonomous function of Pax3 in neural crest cells. We have previously reported that transgenic Splotch mice in which Pax3 expression is reconstituted using the 1.6$\mathrm{kb}$ proximal $\mathrm{Pax} 3$ promoter that is specific for neural tube and neural crest are rescued with respect to cardiac neural crest (but not skeletal muscle) development (46). We analyzed enteric ganglia development in these rescued embryos at E12.5. Enteric ganglia expressing cret were identified in all segments of bowel in transgenic Splotch embryos ( $\mathrm{Sp} / \mathrm{Tg}$, Figure 3j) in contrast to homozygous mutant Splotch littermates, indicating successful rescue of enteric plexus development by expression of Pax3 in neural crest progenitors.

The genes encoding the endothelin receptor $B$ $(E D N R B)$ and its ligand endothelin $3(E D N 3)$ have also been implicated in Hirschsprung's disease $(12,14)$ and are required for distal hindgut ganglia formation. Ednrb is normally expressed by enteric ganglia and also by surrounding mesenchyme (51). In Splotch, ednrb expression is specifically missing in the region where enteric ganglia reside, but it is retained in the surrounding mesenchyme (Figure 3, d and h). Expression of edn 3 in the intestinal mesenchyme is not affected by 

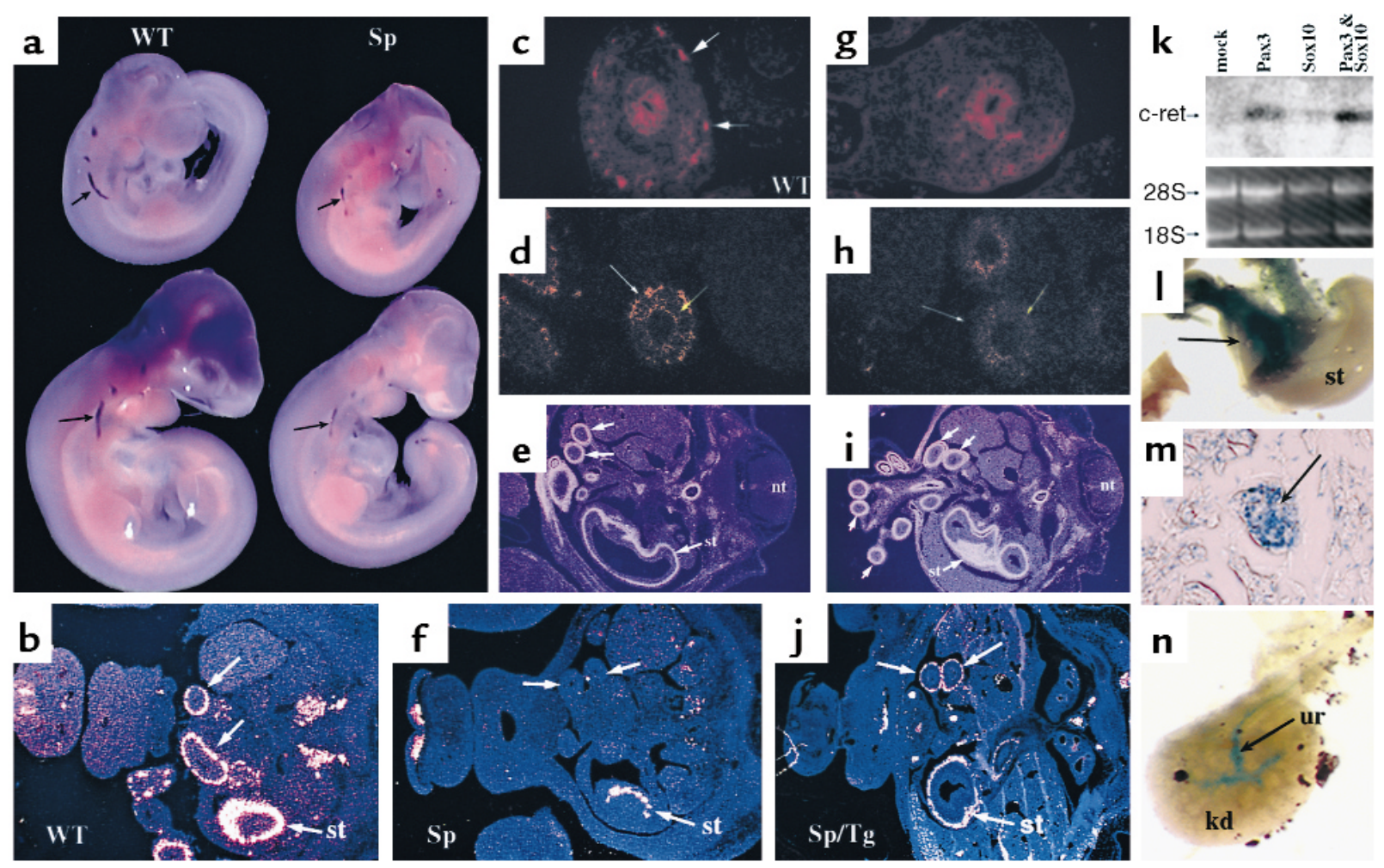

Figure 3

Pax3 is required for normal c-ret expression. (a) Whole-mount in situ hybridization reveals c-ret expression in wild-type (WT) E9.5 (top) and E10 (bottom) embryos. c-ret expression is reduced in Splotch (Sp) embryos (arrows). (b) Radioactive in situ hybridization reveals c-ret expression in the stomach (st) and loops of bowel in wild-type (WT) embryos at E12.5. (f) Expression is missing from intestine distal to the stomach (st) in Splotch (Sp) embryos (arrows). (j) Splotch embryos carrying a transgene that replaces Pax3 expression in neural crest cells $(\mathrm{Sp} / \mathrm{Tg}$ ) have normal c-ret expression in the intestine (arrows). (c and $\mathbf{g}$ ) c-Ret protein expression is evident in the bowel of wild-type E12.5 embryos (arrows, $\mathbf{c}$ ) but not in Splotch intestine $(\mathbf{g})$. ( $\mathbf{d}$ and $\mathbf{h})$ Ednrb mRNA expression is detected by radioactive in situ hybridization in the enteric ganglia of E12.5 wild-type embryos (white arrow, $\mathbf{d}$ ) and in the surrounding mesenchyme (yellow arrow). Ednrb expression is lost the region of normal enteric ganglia formation of Splotch embryos (white arrow, $\mathbf{h}$ ) but retained in surrounding mesenchyme (yellow arrow). (e and i) SM22 $\alpha$ expression, used as a marker of smooth muscle, is normal in wild-type (e) and Splotch (i) embryos. nt, neural tube. (k) Northern analysis of RNA derived from transfected P19 embryocarcinoma cells reveals increased c-ret expression after transfection of Pax3 (lane 2) or Pax3 with Sox10 (lane 4) compared with mock transfection (lane 1). Ethidium bromide-stained gel is shown below to confirm similar loading of RNA in each lane. (I) Transgenic RET-lacZ embryo reveals $\beta$-galactosidase activity in portions of stomach (st, arrow) and intestine. ( $\mathbf{m})$ Section through intestine of sample shown in I reveals $\beta$-galactosidase activity in enteric ganglia. (n) RETlac $Z$ transgenic kidney $(\mathrm{kd})$ reveals $\beta$-galactosidase activity in the ureter (ur).

the absence of Pax3 (data not shown). A smooth muscle marker, SM22 $\alpha$, was expressed normally in Splotch embryos (Figure 3, e and i).

Pax3 and Sox10 activate c-ret. Deficiency of enteric ganglia in Splotch embryos could be a direct result of decreased expression of the c-Ret receptor by enteric ganglia precursors, as c-Ret is required for survival and proliferation of these cells (4-7). Therefore, we tested the ability of Pax3 to activate expression of the c-ret gene in cultured cells by transfecting P19 cells with expression plasmids encoding Pax3 and testing for enhanced c-ret expression. P19 cells can be induced by retinoic acid to express endogenous Pax 3 and can differentiate into neuronal cell types (52). At the same time, we tested the effect of cotransfecting expression plasmids encoding Sox10. Compared with control transfected cells, Pax3 and Pax3 with Sox10 induced significant increases in endogenous c-ret mRNA expression (Figure 3k). Sox10 alone resulted in very slight activation of $c$-ret in this system. RT-PCR experiments (data not shown) confirmed mild upregulation of c-ret after Sox10 transfection.

Using available sequence data, we cloned the human $c-R E T$ promoter to determine whether Pax 3 and Sox 10 are able to specifically regulate transcriptional activity of proximal upstream regulatory regions of the $c$ $R E T$ gene. Transient transgenic mouse embryos containing a $5.1-\mathrm{kb}$ upstream genomic region of $c-R E T$ linked to lac $Z$ were examined at E13.5 to evaluate in vivo activity of this promoter. $\beta$-Galactosidase activity was detected in portions of the stomach and intestine in four transgenic embryos (Figure 3, part l) but not in 26 wild-type littermates. Sections through transgenic intestines revealed $\beta$-galactosidase staining in enteric ganglia (Figure $3 \mathrm{~m}$ ). Nontransgenic intestines processed in parallel never revealed staining. However, 
the staining pattern in transgenic intestines developed slowly and did not encompass the entire endogenous c-ret expression domain, perhaps owing to species differences in regulatory sequences or to enhancer elements located outside of our genomic fragment. We are presently attempting to characterize the murine $c$ - ret promoter to address these possibilities. Transgene expression was consistently detected in the ureter of the developing kidney (Figure 3n), although it did not appear to be confined to the ureteric buds, as is endogenous $c$-ret expression (4). $\beta$-Galactosidase activity was not detected in wild-type littermates.
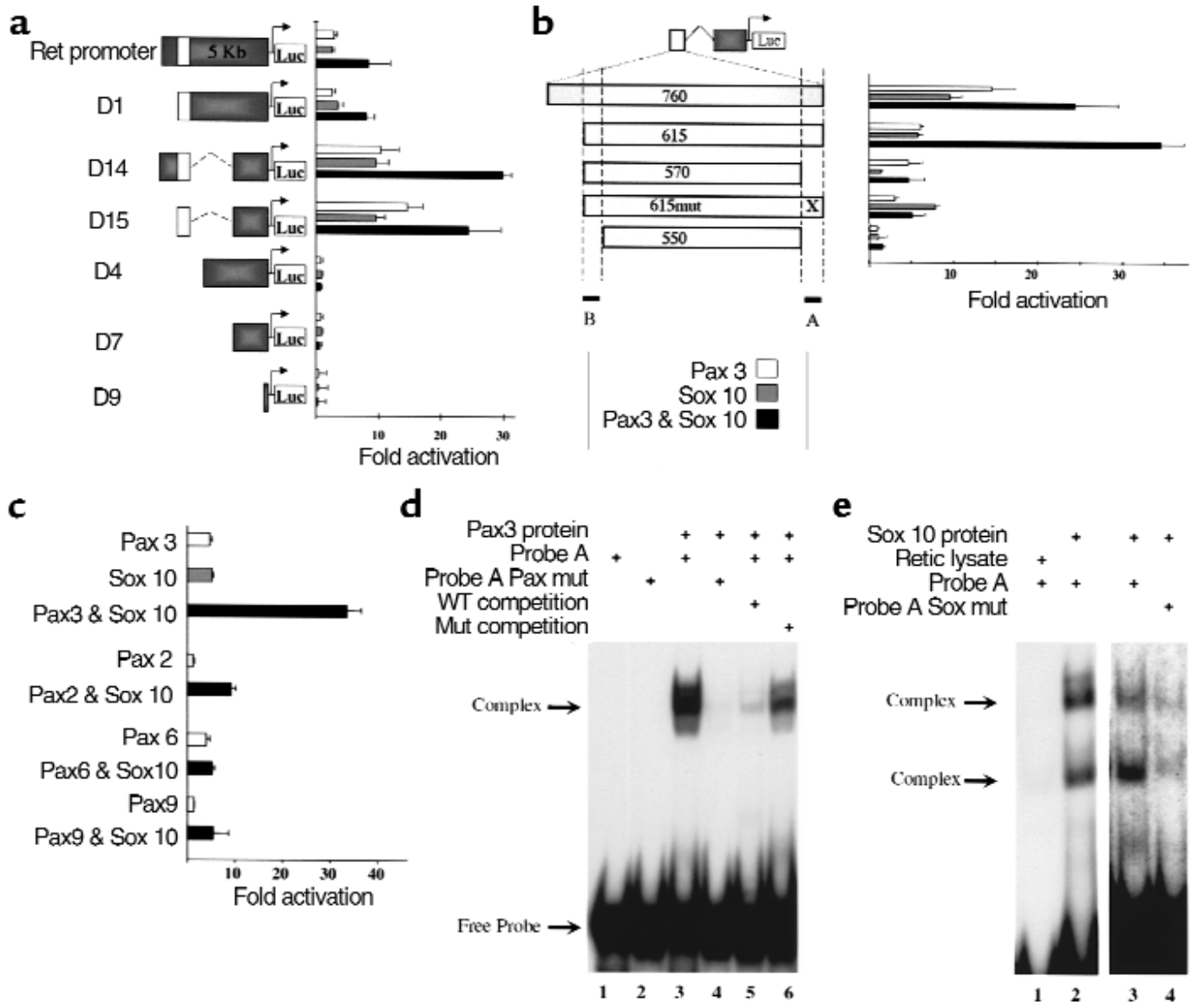

f

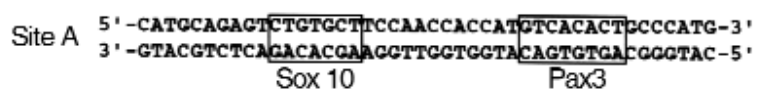

\section{Figure 4}

A Pax3- and Sox10- responsive enhancer in the c-RET gene. (a) Cotransfection of 293T cells with reporter constructs composed of portions of the $c$-RET upstream genomic sequence (see Methods) cloned upstream of a luciferase gene with pCMV-Pax3, pCMV-Sox10, or both reveals a 750-bp enhancer element (open box) and a more proximal repressor element (compare full-length Ret promoter to construct D15). All transfections were normalized for transfection efficiency (see Methods) and are expressed as fold activation compared with transfection without Pax3 or Sox10 (mean \pm SD; $n=12$ for each condition). (b) Further analysis of the 750-bp enhancer was performed by modifying construct D15. Deletion of 45 bp at the $3^{\prime}$ end significantly reduced activity (compare constructs 615 and 570 ). Mutation in a putative Pax3 binding site (indicated by " $X$ " in construct $615 \mathrm{mut}$ ) had a similar effect, although activation by Sox10 alone was not affected by this mutation. Residual Pax 3 responsiveness present in construct 570 is eliminated by deletion of 20 bp at the $5^{\prime}$ end of the enhancer (compare constructs 570 and 550 ). The $3^{\prime}$ and $5^{\prime}$ response elements are indicated by dotted lines and filled bars labeled A and B, respectively. Cotransfection results are expressed as in $\mathbf{a}, n=12$ for each condition. (c) The response of construct 615 (depicted in $\mathbf{b}$ ) is specific for Pax 3 and is activated to a much lesser degree by Pax2, Pax6, and Pax9. Although low-level activation is apparent after cotransfection with Pax 2 or Pax6, no synergistic activation with cotransfected Sox 10 is evident. Cotransfection results are expressed as in $\mathbf{a} ; n=8$ for each condition. Pax 3 and Sox 10 bind to the $c$-RET enhancer. (d) EMSA using a 45-bp probe derived from the $3^{\prime}$ response element in the 750-bp c-RET enhancer (site A in part b) reveals specific binding by Pax3 (lane 3). Pax3 does not bind to a similar probe in which the putative Pax3 binding site has been replaced by an Ascl restriction site (lane 4; and see Methods). Unlabeled competitor wild-type probe is able to compete for binding (lane 5), whereas mutated probe competes far less efficiently (lane 6), indicating binding specificity. (e) Sox10 also binds to site A, and mutation in the putative Sox 10 binding site significantly reduces binding. In vitro translated Sox 10 forms two complexes in EMSA experiments with probe A (lanes 2 and 3 ), whereas the reticulocyte lysate without Sox10 does not bind (lane 1). Mutation in the putative Sox10 binding site (see Methods) reduces Sox10 binding (lane 4). Lanes 1 and 2 are from a different gel than lanes 3 and 4. (f) The sequence of probe A is shown with Sox10 and Pax3 binding sites boxed. 
Identification of Pax3-and Sox10-responsive elements in the $c-R E T$ promoter. In cotransfection assays, Pax 3 and Sox10 were able to activate expression of a $5.1-\mathrm{kb} c$ RET promoter-luciferase reporter both in NIH-3T3 cells (data not shown) and in $293 \mathrm{~T}$ cells, and the presence of Pax3 and Sox10 together produced an approximately ninefold activation (Figure 4a). Analysis of serial deletions of the $c-R E T$ promoter-luciferase reporter construct, depicted in Figure 4a, defined an internal repressor element that, when removed, revealed a 30-fold activation of transcription by Pax3 with Sox10 (construct D14, Figure 4a). The enhancer element responsible for this activity was localized to a 750-bp fragment (construct D15, open box, Figure 4a) that was analyzed further.

A series of deletion constructs (Figure $4 \mathrm{~b}$ and data not shown) composed of portions of the 750-bp enhancer linked to the proximal $c-R E T$ promoter were cotransfected with Pax3, Sox10, or both. These experiments resulted in the identification of a 45-bp element at the $3^{\prime}$ end of the enhancer (site A, Figure 4b) that is required for synergistic activation by Sox 10 and Pax3 (filled bars, Figure 4b; compare constructs 615 and 570). This site contains Sox 10 and Pax3 binding sequences (see below). Mutation of the putative Pax3 paired domain binding site (construct 615mut, Figure $4 \mathrm{~b}$; and see Methods) results in elimination of synergistic activation reproducing the effect of deleting the entire 45 -bp site. Note that construct 615 mut retains responsiveness to Sox 10 (shaded bars, Figure $4 \mathrm{~b}$ ), suggesting that Sox10 binding has not been impaired by the engineered mutation. The residual Pax 3 responsiveness retained by construct 570 (open bar, Figure 4b) is completely eliminated by deletion of $20 \mathrm{bp}$ at the $5^{\prime}$ end of this enhancer (site B, Figure 4b; compare constructs 570 and 550). In summary, cotransfection experiments identified a critical 45-bp element (site A) required for the majority of $c-R E T$ promoter/enhancer activation by Sox 10 and Pax3. Synergistic activation of reporter activity mediated by the $c-R E T$ enhancer was specific for Pax 3 and was not reproduced by cotransfection of Sox10 with other Pax family members including Pax2, Pax6, or Pax9 (Figure 4c).

Pax3 and Sox10 bind to the c-RET enhancer. The region identified as site A in Figure $4 b$, which is responsible for the majority of Pax3 and Sox10 responsiveness, contains a putative Sox 10 binding sequence (CTGTGCT) that contains five of seven nucleotides found in a previously identified Sox10 binding site (CTTTGTT) (26). This site is followed 12 bp downstream by a putative Pax3-paired domain binding site (GTCACACT) that is identical at seven of eight positions to the optimal Pax3-paired domain binding sequence identified in binding site selection assays $(42,53)$. We tested the ability of Pax 3 and Sox 10 to bind to these sequences using EMSAs with endogenous and mutated sequences (see Methods for primer sequences). We used a GST-fusion protein encoding both the paired and homeodomains of Pax 3 , and in vitro translated
Sox10, for these experiments. As shown in Figure 4d, Pax3 is able to bind to site A under stringent binding conditions ( $50 \mathrm{mM} \mathrm{KCl}, 250 \mu \mathrm{g} / \mathrm{ml}$ poly-dIdC), and binding is markedly reduced if the putative paired domain binding site is altered (compare lanes 3 and 4, Figure 4d). Addition of unlabeled wild-type probe effectively competes for specific binding (lane 5, Figure $4 \mathrm{~d}$ ), whereas the unlabeled mutant probe does so far less efficiently (lane 6, Figure 4d). Hence, Pax3 binds to site $\mathrm{A}$ in a sequence specific fashion. In vitro translated Sox10 also binds to site A, producing two shifted complexes in EMSA experiments, suggesting that Sox10 may dimerize on this site (lane 2, Figure $4 e)$. Proteins present in the reticulocyte lysate used to prepare Sox10 did not bind to probe A as demonstrated in lane 1, Figure 4e. Mutation of the putative Sox10 binding sequence significantly reduced Sox 10 binding (compare lanes 4 and 3, Figure 4e). Mutations in the paired domain binding sequence had no effect on Sox10 binding (data not shown). The sequence of probe A and the location of the Sox 10 and Pax3 binding sites identified in these experiments are depicted in Figure 4f. HMG box transcription factors of the Sox10 type are known to bind to the minor groove of DNA (27), whereas Pax proteins bind predominantly to the major groove (54). It is worth noting that the 12bp spacing between Sox 10 and Pax3 sites within the $c$ RET promoter allows for a half turn of the DNA spiral and predicts that both proteins could dock on the same side of DNA, potentially enabling protein-protein interactions. Hence, the $c-R E T$ enhancer contains a 45-bp element that is required for synergistic Pax3 and Sox 10 activation and contains adjacent Pax 3 and Sox10 binding sites.

\section{Discussion}

Our results demonstrate that Pax3-expressing neural crest precursors are fated to become neuronal derivatives of the enteric nervous system. Moreover, analysis of enteric ganglia development in Pax3-deficient Splotch embryos demonstrates that $\mathrm{Pax} 3$ is required for normal development of the enteric plexus. This function of Pax3 is likely to be cell autonomous, as transgenic replacement of Pax 3 in neural crest progenitors is sufficient to rescue enteric ganglia formation (Figure 3j).

Pax3 is normally expressed in premigratory neural crest cells, and expression diminishes during migration (31). Our results indicate that Pax3, together with Sox 10 , can regulate the $c-R E T$ promoter. However, in wild-type embryos, c-ret is strongly expressed by postmigratory enteric ganglia long after Pax3 is extinguished. Message for $c$-ret is also detected in a number of tissues and organs outside of the Pax 3 expression domain, such as the kidney and eye (4). Likewise, not all Pax3-expressing tissues express $c$-ret. Hence, Pax3 is neither necessary nor sufficient for $c$-ret expression under all circumstances and is likely to be important only for initiation or early stages of $c$-ret expression in enteric ganglia precursors. 
We suggest that deficient c-Ret expression in the absence of $\mathrm{Pax} 3$ is related to the ability of $\mathrm{Pax} 3$ to regulate the $c$-RET promoter directly. However, we cannot rule out the possibility that $\operatorname{Pax} 3$ is required for survival or proliferation of enteric ganglia precursors in a manner unrelated to its ability to modulate $c$-ret expression in vitro. These possibilities will prove difficult to differentiate because inappropriate apoptosis of enteric neurons occurs in the absence of $c$-ret $(7,55)$. Thus, it will be difficult to determine whether Pax3 is required for cell survival via c-ret-dependent or -independent pathways. Our demonstration of adjacent Pax 3 and Sox 10 binding sites in the $c$-RET promoter, and the ability of Pax3 and Sox10 to activate $c$-ret expression in P19 cells, suggest that the decrease in c-ret expression evident in Splotch embryos is at least partially attributable to the loss of Pax 3 transcriptional regulation of $c$-ret.

There is precedent for Pax proteins to act in conjunction with other transcriptional regulators in a manner analogous to what we propose here for Pax3 and Sox10. Pax5 is capable of recruiting ETS domain transcription factors to DNA and both factors bind to adjacent sites (56). X-ray crystallographic analysis and computer modeling has suggested that the paired domain binds to the major groove of DNA in a fashion that resembles two linked helix-turn-helix motifs docking on opposite faces of DNA $(54,57)$. Spatial and structural considerations allow for the docking of ETS domain factors to adjacent minor groove positions and suggest possible protein-protein interactions (57). A similar model may apply to Sox and Pax proteins binding to adjacent sites on DNA, and the observation that Sox and Pax binding sites are located 12 bp apart in the $c-R E T$ promoter is consistent with this proposal. To date, we have been unable to demonstrate specific protein-protein interactions between purified Pax3 and Sox10 proteins. However, we have previously demonstrated that the paired domain adopts a stable $\alpha$ helical conformation only upon binding to DNA (58), and hence appropriate stable conformations to allow Pax3-Sox10 interactions may only occur in the presence of specific DNA binding sites.

On the basis of our results, we speculate that other combinations of Pax and Sox transcription factors may synergize to regulate important developmental processes in other systems. For instance, Pax 5 and Sox 4 are both expressed in pre-B cells (59-61), and inactivation of either gene in the mouse results in arrest of Bcell development at similar or identical stages $(60,61)$. Pax 5 binds to and activates the promoter for the CD19 cell-surface receptor (62), and it would be interesting to test whether Sox4, which is closely related to Sox10, can modify the ability of Pax 5 to activate CD19 or other downstream targets in B cells.

In summary, in both humans and mice, PAX3, SOX10, and $c$-RET mutations result in neural crest-related defects. In some cases, there is phenotypic overlap between the resulting syndromes. Here we provide evidence that Pax3-expressing neural crest precursors give rise to enteric ganglia and that $\mathrm{Pax} 3$ is required for this developmental process. Pax3 functions with Sox 10 to modulate expression of $c-R E T$, and Pax 3 and Sox 10 bind to adjacent sites within a $c-R E T$ enhancer. Pax3, Sox 10, and c-Ret are components of a molecular cascade regulating neural crest development.

\section{Acknowledgments}

We are grateful to R. Mass, H. Peters, M. SouthardSmith, and W. Pavan for providing plasmids. This work was supported by NIH grants RO1HL62974, RO1HL61475, RO1DK57050 and a pilot project of Center grant P30DK50306 to JAE.

1. LeDouarin, N.M. 1982. The neural crest. Cambridge University Press. London, United Kingdom. 259 pp.

2. McKusick, V.A. 1998. Online mendelian inheritance in man, $\mathrm{OMIM}^{\mathrm{TM}}$. http://www.ncbi.nlm.nih.gov/Omim. Johns Hopkins University and National Center for Biotechnology Information, National Library of Medicine. Baltimore, Maryland, USA, and Bethesda, Maryland, USA.

3. Gershon, M.D. 1999. Disorders of enteric neuronal development: insights from transgenic mice. Am. J. Physiol. 277:G262-G267.

4. Pachnis, V., Mankoo, B., and Costantini, F. 1993. Expression of the cret proto-oncogene during mouse embryogenesis. Development. 119:1005-1017.

5. Romeo, G., et al. 1994. Point mutations affecting the tyrosine kinase domain of the RET proto-oncogene in Hirschsprung's disease. Nature. 367:377-378.

6. Edery, P., et al. 1994. Mutations of the RET proto-oncogene in Hirschsprung's disease. Nature. 367:378-380.

7. Durbec, P.L., Larsson-Blomberg, L.B., Schuchardt, A., Costantini, F., and Pachnis, V. 1996. Common origin and developmental dependence on c-ret of subsets of enteric and sympathetic neuroblasts. Development. 122:349-358.

8. Durbec, P., et al. 1996. GDNF signalling through the Ret receptor tyrosine kinase. Nature. 381:789-793.

9. Moore, M.W., et al. 1996. Renal and neuronal abnormalities in mice lacking GDNF. Nature. 382:76-79.

10. Pichel, J.G., et al. 1996. Defects in enteric innervation and kidney development in mice lacking GDNF. Nature. 382:73-76.

11. Sanchez, M.P., et al. 1996. Renal agenesis and the absence of enteric neurons in mice lacking GDNF. Nature. 382:70-73.

12. Baynash, A.G., et al. 1994. Interaction of endothelin-3 with endothelin$B$ receptor is essential for development of epidermal melanocytes and enteric neurons. Cell. 79:1277-1285.

13. Hosoda, K., et al. 1994. Targeted and natural (piebald-lethal) mutations of endothelin-B receptor gene produce megacolon associated with spotted coat color in mice. Cell. 79:1267-1276.

14. Puffenberger, E.G., et al. 1994. A missense mutation of the endothelin$\mathrm{B}$ receptor gene in multigenic Hirschsprung's disease. Cell. 79:1257-1266.

15. Gariepy, C.E., Cass, D.T., and Yanagisawa, M. 1996. Null mutation of endothelin receptor type B gene in spotting lethal rats causes aganglionic megacolon and white coat color. Proc. Natl. Acad. Sci. USA. 93:867-872.

16. Edery, P., et al. 1996. Mutation of the endothelin-3 gene in the Waardenburg-Hirschsprung disease (Shah-Waardenburg syndrome). Nat. Genet. 12:442-444.

17. Pattyn, A., Morin, X., Cremer, H., Goridis, C., and Brunet, J.F. 1999. The homeobox gene Phox $2 \mathrm{~b}$ is essential for the development of autonomic neural crest derivatives. Nature. 399:366-370.

18. Southard-Smith, E.M., Kos, L., and Pavan, W.J. 1998. Sox10 mutation disrupts neural crest development in Dom Hirschsprung mouse model. Nat. Genet. 18:60-64.

19. Pingault, V., et al. 1998. SOX10 mutations in patients with Waardenburg-Hirschsprung disease. Nat. Genet. 18:171-173.

20. Herbarth, B., et al. 1998. Mutation of the Sry-related Sox10 gene in Dominant megacolon, a mouse model for human Hirschsprung disease. Proc. Natl. Acad. Sci. USA. 95:5161-5165.

21. Lo, L.C., Johnson, J.E., Wuenschell, C.W., Saito, T., and Anderson, D.J. 1991. Mammalian achaete-scute homolog 1 is transiently expressed by spatially restricted subsets of early neuroepithelial and neural crest cells. Genes Dev. 5:1524-1537.

22. Guillemot, F., et al. 1993. Mammalian achaete-scute homolog 1 is required for the early development of olfactory and autonomic neurons. Cell. 75:463-476. 
23. Blaugrund, E., et al. 1996. Distinct subpopulations of enteric neuronal progenitors defined by time of development, sympathoadrenal lineage markers and Mash-1-dependence. Development. 122:309-320.

24. Hofstra, R.M., et al. 1996. A homozygous mutation in the endothelin3 gene associated with a combined Waardenburg type 2 and Hirschsprung phenotype (Shah-Waardenburg syndrome). Nat. Genet. 12:445-447.

25. Attie, T., et al. 1995. Mutation of the endothelin-receptor B gene in Waardenburg-Hirschsprung disease. Hum. Mol. Genet. 4:2407-2409.

26. Kuhlbrodt, K., Herbarth, B., Sock, E., Hermans-Borgmeyer, I., and Wegner, M. 1998. Sox10, a novel transcriptional modulator in glial cells. $J$. Neurosci. 18:237-250.

27. Giese, K., Cox, J., and Grosschedl, R. 1992. The HMG domain of lymphoid enhancer factor 1 bends DNA and facilitates assembly of functional nucleoprotein structures. Cell. 69:185-195.

28. Grosschedl, R., Giese, K., and Pagel, J. 1994. HMG domain proteins: architectural elements in the assembly of nucleoprotein structures. Trends Genet. 10:94-100.

29. Baldwin, C.T., Hoth, C.F., Amos, J.A., da Silva, E.O., and Milunsky, A. 1992. An exonic mutation in the HuP2 paired domain gene causes Waardenburg's syndrome. Nature. 355:637-638.

30. Tassabehji, M., et al. 1992. Waardenburg's syndrome patients have mutations in the human homologue of the Pax-3 paired box gene. Nature. 355:635-636.

31. Goulding, M.D., Chalepakis, G., Deutsch, U., Erselius, J.R., and Gruss, P. 1991. Pax-3, a novel murine DNA binding protein expressed during early neurogenesis. EMBO J. 10:1135-1147.

32. Ayme, S., and Philip, N. 1995. Possible homozygous Waardenburg syndrome in a fetus with exencephaly. Am. J. Med. Genet. 59:263-265.

33. Tassabehji, M., Newton, V., and Read, A. 1994. Waardenburg syndrome type 2 caused by mutations in the human microphthalmia (MITF) gene. Nat. Genet. 8:251-255.

34. Watanabe, A., Takeda, K., Ploplis, B., and Tachibana, M. 1998. Epistatic relationship between Waardenburg syndrome genes MITF and PAX3 Nat. Genet. 18:283-286.

35. Epstein, D.J., Vekemans, M., and Gros, P. 1991. Splotch (Sp2H), a mutation affecting development of the mouse neural tube, shows a deletion within the paired homeodomain of Pax-3. Cell. 67:767-774.

36. Epstein, D.J., Vogan, K.J., Trasler, D.G., and Gros, P. 1993. A mutation within intron 3 of the Pax-3 gene produces aberrantly spliced mRNA transcripts in the splotch (Sp) mouse mutant. Proc. Natl. Acad. Sci. USA 90:532-536.

37. Auerbach, R. 1954. Analysis of the developmental effects of a lethal mutation in the house mouse. J. Exp. Zool. 127:305-329.

38. Li, J., Chen, F., and Epstein, J.A. 2000. Neural crest expression of Cre recombinase directed by the proximal Pax3 promoter in transgenic mice. Genesis. 26:162-164.

39. Tajbakhsh, S., Rocancourt, D., Cossu, G., and Buckingham, M. 1997. Redefining the genetic hierarchies controlling skeletal myogenesis: Pax3 and myf-5 act upstream of myoD. Cell. 89:127-138.

40. Wawersik, S., and Epstein, J.A. 2000. Gene expression analysis by in situ hybridization. In Methods in molecular biology. Volume 137. Developmental biology protocols. R.S. Tuan and C.W. Lo, editors. Humana Press Inc. Totowa, New Jersey, USA. 87-96

41. Riddle, R.D., Johnson, R.L., Laufer, E., and Tabin, C. 1993. Sonic hedgehog mediates the polarizing activity of the ZPA. Cell. 75:1401-1416.

42. Epstein, J.A., Shapiro, D.N., Cheng, J., Lam, P.Y., and Maas, R.L. 1996. Pax3 modulates expression of the c-Met receptor during limb muscle development. Proc. Natl. Acad. Sci. USA. 93:4213-4218.
43. Chalepakis, G., Jones, F.S., Edelman, G.M., and Gruss, P. 1994. Pax-3 contains domains for transcription activation and transcription inhibition. Proc. Natl. Acad. Sci. USA. 91:12745-12749.

44. Epstein, J.A., et al. 1994. Two independent and interactive DNA binding subdomains of the PAX6 paired domain are regulated by alternative splicing. Genes Dev. 8:2022-2034.

45. Epstein, J.A., Lam, P., Jepeal, L., Maas, R.L., and Shapiro, D.N. 1995. Pax3 inhibits myogenic differentiation of cultured myoblast cells. J. Biol. Chem. 270:11719-11722.

46. Li, J., Liu, K.C., Jin, F., Lu, M.M., and Epstein, J.A. 1999. Transgenic rescue of congenital heart disease and spina bifida in Splotch mice. Development. 126:2495-2503.

47. Epstein, J.A., et al. 2000. Migration of cardiac neural crest cells in Splotch embryos. Development. 127:1869-1878.

48. Soriano, P. 1999. Generalized lacZ expression with the ROSA26 Cre reporter strain. Nat. Genet. 21:70-71.

49. Natoli, T.A., Ellsworth, M.K., Wu, C., Gross, K.W., and Pruitt, S.C. 1997. Positive and negative DNA sequence elements are required to establish the pattern of Pax3 expression. Development. 124:617-626.

50. Waldo, K.L., Lo, C.W., and Kirby, M.L. 1999. Connexin 43 expression reflects neural crest patterns during cardiovascular development. Dev. Biol. 208:307-323.

51. Wu, J.J., Chen, J.X., Rothman, T.P., and Gershon, M.D. 1999. Inhibition of in vitro enteric neuronal development by endothelin-3: mediation by endothelin B receptors. Development. 126:1161-1173.

52. Pruitt, S.C. 1992. Expression of Pax-3- and neuroectoderm-inducing activities during differentiation of P19 embryonal carcinoma cells. Development. 116:573-583.

53. Chalepakis, G., and Gruss, P. 1995. Identification of DNA recognition sequences for the Pax3 paired domain. Gene. 162:267-270.

54. Xu, W., Rould, M.A., Jun, S., Desplan, C., and Pabo, C.O. 1995. Crystal structure of a paired domain-DNA complex at 2.5 A resolution reveals structural basis for Pax developmental mutations. Cell. 80:639-650.

55. Taraviras, S., et al. 1999. Signalling by the RET receptor tyrosine kinase and its role in the development of the mammalian enteric nervous system. Development. 126:2785-2797.

56. Fitzsimmons, D., et al. 1996. Pax-5 (BSAP) recruits Ets proto-oncogene family proteins to form functional ternary complexes on a B-cell-specific promoter. Genes Dev. 10:2198-2211.

57. Xu, H.E., et al. 1999. Crystal structure of the human Pax6 paired domain-DNA complex reveals specific roles for the linker region and carboxy-terminal subdomain in DNA binding. Genes Dev. 13:1263-1275.

58. Epstein, J., Cai, J., Glaser, T., Jepeal, L., and Maas, R. 1994. Identification of a Pax paired domain recognition sequence and evidence for DNAdependent conformational changes. J. Biol. Chem. 269:8355-8361.

59. Adams, B., et al. 1992. Pax-5 encodes the transcription factor BSAP and is expressed in B lymphocytes, the developing CNS, and adult testis. Genes Dev. 6:1589-1607.

60. Urbanek, P., Wang, X.Q., Fetka, I., Wagner, E.F., and Busslinger, M. 1994. Complete block of early B cell differentiation and altered patterning of the posterior midbrain in mice lacking Pax5/BSAP. Cell. 79:901-912.

61. Schilham, M.W., et al. 1996. Defects in cardiac outflow tract formation and pro-B-lymphocyte expansion in mice lacking Sox-4. Nature. 380:711-714.

62. Kozmik, Z., Wang, S., Dorfler, P., Adams, B., and Busslinger, M. 1992. The promoter of the CD19 gene is a target for the B-cell-specific transcription factor BSAP. Mol. Cell. Biol. 12:2662-2672. 